\title{
Implantación de la política en salud materna en la República de Chile y Perú: una observación desde la dimensión de gestión
}

\author{
Keller Sánchez
}

Universidad de Chile

\section{Resumen}

Con el objetivo de entender la alta variabilidad en la mortalidad materna en América Latina y el Caribe, esta investigación analiza los casos de la República de Perú y la República de Chile, utilizando una metodología con enfoque cualitativo de tipo descriptivo. Se observó que en materia de diseño organizacional los ministerios de salud que cuentan con una estructura organizacional segmentada y fragmentada, presentan dificultades para articular sus acciones, no logrando las coberturas para la población, generando una mayor inequidad en el acceso a atención médica. En este mismo sentido se observan diferencias en el énfasis de la política, en que para el caso chileno se concentra en promoción y prevención mientras que en el caso peruano se concentra más en la parte recuperativa.

Palabras clave: Mortalidad materna, diseño organizacional, salud pública.

Implantation of maternal health policy in the Republic of Chile and Peru: an observation from the management dimension

\begin{abstract}
This research aims to understand the high variability in maternal mortality in Latin America and the Caribbean. For that the paper analyzes the cases of the Republic of Peru and the Republic of Chile, using a methodology descriptive qualitative approach. It was observed that in organizational design, health ministries that have a segmented and fragmented organizational structure, have difficulties in articulating their actions, not achieving coverage for the population, generating greater inequality in access to health care. In this sense differences in policy emphasis were observed, while the chilean case focuses on promotion and prevention, in the Peruvian case it focuses more on the recuperative part.
\end{abstract}

Keywords: Maternal mortality, organizational design, public health. 


\section{Introducción}

La política sanitaria tiene tres objetivos que deben ser ponderados: maximizar la salud, reducir las desigualdades en salud y favorecer a aquellos que lo necesitan; aumentando la calidad de vida y la eficiencia, teniendo como referentes fundamentales la equidad y la ética, en el marco del contrato social de los profesionales, los gestores y los políticos sanitarios (Gérvas y Pérez, 2011).

Las políticas reguladoras pueden definir acreditaciones profesionales, establecer controles de precios para los bienes y servicios, determinar criterios de calidad, seguridad y eficacia para los servicios de la salud, y abordar cuestiones de regulación social, tales como las relacionadas con la seguridad social y ocupacional, la inmunización, los alimentos y medicamentos, y la contaminación ambiental (Organización Panamericana de la Salud, 2007).

En consecuencia, llevar a cabo transformaciones que refuercen la importancia de la estructura del sistema de salud como institución social central, capaz de crear oportunidades diferenciales en el diseño y la prestación de bienes y servicios, requiere un abordaje sistémico de las políticas públicas y de su formulación. La política pública puede entenderse como un conjunto de decisiones bien fundamentadas, generadas por cualquiera de las ramas y en todos los niveles del gobierno, y enmarcadas en un conjunto de normativas. Las políticas públicas comunican objetivos, medios, estrategias y reglas para la toma de decisiones, utilizados en la gestión pública y la legislación.

\section{Gestión en salud: El componente es- tructural de la política de salud}

La medicina tiene un rol social, que puede ser definido al menos a través de tres dimensiones. En primer lugar, cuando una persona percibe algún problema de salud no acude a la medicina empresarial sino a la "medicina". En segundo lugar, los gobiernos no delegan la responsabilidad por la habilitación y acreditación profesional a la industria de la atención médica sino a la profesión médica. Por último, la industria de la atención médica, donde la medicina siempre ha señalado el camino y la organización empresarial no ha hecho más que seguirlo (Studin, 2000).

En síntesis, la problemática de la gestión en salud causa una tensión entre dos formas de pensar, por un lado el de la medicina y por el otro el de la gestión o administración de la organización. Se plantea a continuación dos distinciones conceptua- les que permiten identificar con mayor precisión las particularidades de la gestión en salud. Se trata, en primer lugar, de una distinción de orden y en segundo lugar de una distinción de grado. La primera involucra identificar tres componentes diferentes de todo servicio o sistemas de salud, cada uno de los cuales circunscribe decisiones específicas. La segunda distingue tres niveles de decisión en la gestión de salud.

\section{Componentes de un sistema de salud}

Se pueden distinguir tres componentes de un servicio de salud: político, económico y técnico. Cada uno de ellos involucra un conjunto de definiciones particulares, a cuestiones específicas. Sin embargo, existen también problemas comunes como el conjunto de combinaciones. De esta manera se puede pensar a los servicios de salud como al conjunto de combinaciones que admite la interacción de tres conjuntos: a) uno político, al que llamamos modelo de gestión, b) uno económico, al que llamamos modelo financiero, c) uno técnico, al que llamamos modelo de atención o modelo asistencial.

Respecto a lo planteado por Ortún (1996), los servicios de salud deben ser capaces de garantizar la articulación de las tres dimensiones, teniendo así un objetivo común, encarado desde las diferentes dimensiones, para así contar con un trabajo articulado y encaminado en la misma dirección cual es minimizar las MM.

\section{Niveles de gestión en salud}

Rubio (1996) ha formulado una taxonomía que distingue tres niveles de gestión en salud: Un nivel de macrogestión, que involucra la intervención del Estado para corregir las fallas del mercado en salud y mejorar el bienestar social. Un ambiente de mesogestión o gestión institucional, que involucra centros, hospitales, aseguradoras y otros establecimientos de salud. Un ambiente microgestión o gestión clínica donde la mayor responsabilidad cabe a los profesionales.

Finalmente, las particularidades propias que tiene el sistema de salud, desde sus diferentes componentes y niveles de gestión, hacen que la implementación de las políticas de salud materna, tenga desafíos frecuentes como la coordinación, negociación, etc., imposibilitando muchas veces lograr los objetivos planificados. Es justamente aquí donde la gestión adquiere importancia, al articular diferentes actores que poseen diferentes intereses. 


\section{Política en salud materna}

A pesar de los avances logrados en los años posteriores a la Conferencia Internacional sobre la Población y el Desarrollo, realizada en El Cairo en 1994, y la Cuarta Conferencia Mundial sobre la Mujer, celebrada en Beijing en 1995, el impacto de las acciones para mejorar la Salud Sexual y Reproductiva (SSR) a juicio de la Organización Panamericana de la Salud (2007) es muy débil. Esto se debe a que no se ha resuelto cómo disminuir la brecha de inequidad, ni cómo dar apoyo a los países, a sus líderes y a la comunidad para cambiar las disparidades en información y servicios, con el fin de obtener mejores resultados en la distribución de bienes de salud. Al considerar la salud sexual y reproductiva, la Conferencia Internacional se ha pronunciado sobre varios aspectos clave. Primero, todos los individuos tienen derecho a un completo estado de bienestar físico, mental y social, y no solo la ausencia de enfermedades, en todos los aspectos relacionados con el sistema reproductivo, sus funciones o procesos. La salud reproductiva, por lo tanto, implica que las personas puedan disfrutar de una vida sexual satisfactoria, segura y responsable, así como la capacidad para reproducirse y la libertad de decidir si se reproducen, cuándo y con qué frecuencia. Lo anterior conlleva el derecho de hombres y mujeres a la información y al acceso a métodos anticonceptivos seguros, efectivos, aceptables y asequibles financieramente; el derecho a servicios de salud de calidad para la atención del embarazo, el parto y el recién nacido; el derecho a servicios de atención de infecciones de transmisión sexual, incluyendo $\mathrm{VIH} /$ sida, y a la prevención del cáncer del sistema reproductivo, de discapacidades generadas en el climaterio, y de la violencia sexual (Organización Panamericana de la Salud, 2007).

Así pues, las políticas públicas de salud sexual y reproductiva están dirigidas hacia la reducción de los decesos maternos, para lo que deben ser diseñadas en conjunto con otras políticas que busquen reducir y eliminar las desigualdades, así como los obstáculos institucionales y estructurales diversos (Comisión Económica para América Latina y el Caribe et al., 2009).

$\mathrm{Al}$ mismo tiempo, se reconoce que la mayoría de los casos de mortalidad y morbilidad materna son prevenibles, y que la mortalidad y morbilidad maternas prevenibles suponen un problema de salud, desarrollo y derechos humanos que también exige la promoción y protección efectiva de los derechos humanos de las mujeres y las niñas, en particular su derecho a la vida, a ser iguales en dignidad, a la educación, a ser libres para buscar, recibir y difundir información, a gozar de los beneficios del progreso científico, a estar a salvo de la discriminación y a disfrutar del nivel más alto posible de salud física y mental, incluida la salud sexual y reproductiva; por lo que es relevante que los Estados renueven su compromiso político de eliminar la mortalidad y morbilidad maternas prevenibles en los planos local, nacional, regional e internacional y que redoblen sus esfuerzos por lograr el cumplimiento pleno y efectivo de sus obligaciones en materia de derechos humanos (Naciones Unidas, 2000).

\section{El problema de la mortalidad materna}

La Mortalidad Materna representa un grave problema de salud pública y de derechos humanos. Sus causas son evitables y es la máxima expresión de inequidad e injusticia social, ya que es en los países de menor desarrollo económico donde existen las cifras más altas de muertes maternas, pues son las mujeres pobres las que tienen mayor riesgo de morir por embarazo, parto o puerperio, al carecer de poder de decisión, aún en el seno de sus propias familias (Organización Mundial de la Salud et al., 2003).

El indicador utilizado para medir la mortalidad materna, es la razón de mortalidad materna la cual ha sido definida por un acuerdo internacional como el número de muertes maternas en un año por 100 mil nacidos vivos. La tasa expresa el número de muertes maternas en un año por 100 mil mujeres en edad reproductiva (15-49 años), sin embargo convencionalmente a esta primera la denominamos tasa (Organización Panamericana de la Salud, 2007).

Entre los factores vinculados con las complicaciones del embarazo y el parto se pueden distinguir dos tipos: uno de origen endógeno, relacionado con las condiciones biológicas de la madre; y un componente exógeno, que depende en gran medida de factores asociados a condiciones del medio ambiente social y de la calidad, acceso, oportunidad y tipo de atención médica recibida (Organización Mundial de la Salud et al., 1999). La reducción de la mortalidad materna depende en gran parte de la existencia de establecimientos de salud que funcionen de manera efectiva. La pérdida de la vida de una mujer en el parto es, en última instancia, el mayor fracaso del sistema de salud. Este fracaso, que se repite a razón de uno por minuto en el mundo en desarrollo, causa irrevocables pérdidas y viola el derecho de la mujer a la vida (Comisión Económica para América Latina y el Caribe, 2004).

Según el de Administración y Políticas de Salud (1997) para disminuir la mortalidad materna es necesario un fortalecimiento adecuado de los sistemas de salud, que se cuente con los recursos necesarios 
para la atención de las pacientes obstétricas graves y la coordinación adecuada entre las autoridades de las instituciones de salud y los prestadores del servicio, para dar una atención oportuna y adecuada (de Administración y Políticas de Salud, 1997). Es necesario sin embargo, la intervención interdisciplinaria (políticos, autoridades gubernamentales, educativas, de instituciones de salud, etcétera) para lograr reducir la pobreza extrema, alcanzar una educación primaria universal, nacimientos asistidos por personal profesional, acceso a servicios de salud de calidad, planificación familiar al alcance de todos y mejorar la condición social de la mujer a través del esfuerzo conjunto (Organización Mundial de la Salud et al., 2003; OPS, 2006)

\section{Objetivos de estudio}

El objetivo general de esta investigación es observar el proceso de implantación de la Política en Salud Materna durante el período 2000 a 2010 y establecer propuestas de mejora para la implementación de la política materna. Para ello se propuso determinar las diferencias del proceso de implementación de la política materna, desde su dimensión de gestión; describir las estrategias ejecutadas durante el proceso de implementación de la política materna; y, finalmente, generar propuestas de política pública que contribuyan a la mejora del proceso de implementación de la política materna desde su dimensión de gestión.

El estudio seleccionó dos casos de implementación de política materna en América Latina, utilizando como criterios generales el comportamiento de los casos en lo que se refiere a cifras de MM, en este sentido se buscaron casos opuestos en que uno tuviera una alta incidencia y el otro una baja incidencia de MM. Por otra parte, un criterio que obedece a aspectos de conveniencia, asociado principalmente a la disponibilidad de información en cuanto a política pública de salud y datos estadísticos de MM. De esta forma se seleccionaron los casos de la República de Perú y de la República Chile cuyas cifras y evolución de MM se presentan en el capítulo de resultados.

\section{Metodología}

El presente estudio se enmarca en un enfoque cualitativo de tipo descriptivo, en tanto se aproxima a identificar y determinar las diferencias de la implantación de la política en salud materna en los casos seleccionados. La tipología de estudio de caso, según el modelo desarrollado por Yin (2013), corresponde a un tipo "incrustado" en que se consideran múltiples unidades de análisis, como son los casos de la República de Perú y Chile.

La estrategia de comparación de ambos casos se centra en un modelo compuesto por varias dimensiones que dan cuenta de una dimensión mayor denominada procesos de implementación desde la dimensión de gestión. Asimismo, se plantea el mapa de análisis en un contexto general para dar cuenta de las variables y los tópicos que se abordaron en el proceso de estudio.

La estrategia de levantamiento de datos para este estudio se realizó mediante la revisión documental, seleccionando documentos oficiales de los Ministerios de Salud de ambos países; asimismo documentos que presentaban una mirada general del proceso de implementación de la política en salud de organizaciones internacionales como la OPS, OMS, CEPAL, entre otros, que abordan el tema de salud materna, haciendo hincapié en las diferencias que muestran los países de la región. El instrumento de recolección de datos que se empleó en el estudio fue la matriz documental, la cual se deriva de la tabla anterior agregando a cada dimensión una o más variables que permitieron medir y/o acceder a aspecto específico de cada caso seleccionado. Los documentos seleccionados fueron en base a un muestreo intencionado por criterios. Los criterios de elección documentaria se apoyan en reportes e informes de expertos e instituciones internacionales, como la Organización Mundial de la Salud (OMS). Así mismo se utilizó documentos de la Organización Panamericana de la Salud (OPS). También se incluirán en el análisis documentos de la Comisión Económica para América Latina y el Caribe (CEPAL). Por último, se seleccionaron informes de los Ministerios de Salud de cada país en estudio. Una vez recolectada la información se analizó a través de la técnica de análisis de contenido, lo cual consiste en un método de observación, el que permitió realizar observaciones sobre conjuntos de información, con miras a producir resultados en distintos niveles de profundidad de acuerdo a la construcción e intención de las propias dimensiones (Pérez Serrano, 2002).

Es necesario mencionar que los documentos analizados para el presente estudio tenían un enfoque general de la implementación de las políticas en salud, en sus diferentes ámbitos de acción tanto desde la etapa de perinatal hasta la adultez. Ello fue obstáculo para lograr una visión detallada de la política materna, dado que ésta no era abordada de forma específica. Por otro lado, la disponibilidad de documentos en los portales web de algunas instituciones no estaba actualizada, impidiendo tener acceso a fuentes documentales de mayor calidad. 
Tabla 1: Mapa de análisis

\begin{tabular}{|c|c|c|}
\hline Dimennioues & Variables & Topicen \\
\hline \multirow{4}{*}{$\begin{array}{l}\text { Orieataciones } \\
\text { generales de } \\
\text { política }\end{array}$} & \multirow[t]{3}{*}{ Historia } & Comprouniso gubernameatal. \\
\hline & & Reconocianiento constítucional del acceso universal en salud. \\
\hline & & Vinculación con los cojetivos del milenio. \\
\hline & Cifras de MM & Reduccióa sestenida de la MM \\
\hline \multirow{5}{*}{$\begin{array}{l}\text { Diseñe } \\
\text { organizacional }\end{array}$} & \multirow{2}{*}{$\begin{array}{l}\text { Estructura } \\
\text { orgazizacional }\end{array}$} & Estructura segmentado. \\
\hline & & Estructura fragmeatado. \\
\hline & $\begin{array}{l}\text { Focalizacibo de grupo } \\
\text { vulnerable }\end{array}$ & $\begin{array}{l}\text { Identificacion de grupos vulnerables y con enfasis en los pueblos } \\
\text { indigenas }\end{array}$ \\
\hline & \multirow{2}{*}{$\begin{array}{l}\text { Gestice en politica } \\
\text { materna }\end{array}$} & Focalizació en APS. \\
\hline & & Focalizaciba en aseguramicatos universal. \\
\hline \multirow[t]{8}{*}{ Conunicación } & \multirow[t]{2}{*}{ Intrascctorial } & Alta burocratización. \\
\hline & & SEstema único de información. \\
\hline & \multirow[t]{2}{*}{ Intersectorial } & Desarticulació instisucional. \\
\hline & & Duplicidad de funciones. \\
\hline & Fuente de informacion & Regisitro de dasos coatiables. \\
\hline & Accesibilidad & Sistema de referencia y contrareferencia de atenciba y tratalniento. \\
\hline & \multirow[t]{2}{*}{ Comunicación sanitaria } & Centradas en promocion y preveación. \\
\hline & & Centrada en unateraidad saludable y segura. \\
\hline \multirow[t]{13}{*}{ Recursos } & \multirow[t]{8}{*}{ Humanos } & Sistema de capacitación. \\
\hline & & Sistema de apoyo a ha gestion santaria. \\
\hline & & Incremenso de personal obstétrica. \\
\hline & & Exodo de profesiceales de la salud. \\
\hline & & Emigración de personal calificado. \\
\hline & & Disponibulidad de personal capacitado en las regiones. \\
\hline & & Alta rotación de profesionales de la salud. \\
\hline & & Flexible laboral del personal de salud. \\
\hline & \multirow[t]{5}{*}{ Financieros } & Subsistemas coo diversas formas de financianienso y prestaciones. \\
\hline & & Asigaación de gasto a actividades de promocion y preveacion. \\
\hline & & Finasciamieato publico reducido. \\
\hline & & $\begin{array}{l}\text { Incremenso progresivo de presupuesto asignado a la ateacion } \\
\text { materna. }\end{array}$ \\
\hline & & $\begin{array}{l}\text { Recursos para mantenimicato de infraestructura y reposicióa de } \\
\text { equipos médicos en los establecimientos. }\end{array}$ \\
\hline \multirow[t]{4}{*}{ Negociacion } & \multirow[t]{2}{*}{ Intrasectorial } & Prounueve la concertacion sockorial y tambien Elentsectorial. \\
\hline & & Resolucióa de conflictos laborales. \\
\hline & \multirow[t]{2}{*}{ Intersectorial } & $\begin{array}{l}\text { Esfuerzo con otros sectores, para aunpliar la cobertura de ateacion } \\
\text { prenatal y del parto iestitucional. }\end{array}$ \\
\hline & & $\begin{array}{l}\text { Fortaleciniento de la promocion de salud ea las Instituciones } \\
\text { Educativas. }\end{array}$ \\
\hline \multirow[t]{8}{*}{ Inmovació } & \multirow[t]{4}{*}{ Techologica } & $\begin{array}{l}\text { Renovación de los equipos e intervenciones de seporte técnico } \\
\text { informátios. }\end{array}$ \\
\hline & & $\begin{array}{l}\text { Creacion de unidades u organizaciooes dedicadas a la evaluacion } \\
\text { de las tecnologias de salud. }\end{array}$ \\
\hline & & Insplantacion del sistema integrado de gestion adninistrativa. \\
\hline & & $\begin{array}{l}\text { Sistema de información en salud con implementacion de ficha } \\
\text { clinica electrónica. }\end{array}$ \\
\hline & \multirow[t]{2}{*}{$\begin{array}{l}\text { Insumos y } \\
\text { medicamentos }\end{array}$} & $\begin{array}{l}\text { Programación de medicamentos e insumos estrategicos a aivel } \\
\text { aacional. }\end{array}$ \\
\hline & & Politica Nacional de Medicamentos, enumarcada en el uso racional. \\
\hline & Investigacion & SEstema aacional de investigacion ea salud estructurado. \\
\hline & Politica & sovacióa ea salud materna, oricatada al usuario. \\
\hline
\end{tabular}

Fuente: Elaboración propia, a partir de la revisión documental 


\section{Resultados}

\section{Política pública en salud materna en la República de Perú}

\section{Primer momento histórico}

En este primer momento se desarrolla el Acuerdo Nacional, formulado e implementado en el gobierno de Alejandro Toledo Manrique (período, entre el 28 de julio de 2001 y el 28 de julio de 2006), que buscaba alcanzar el bienestar de las personas, así como el desarrollo humano y solidario en el país, con representantes de las organizaciones políticas, religiosas, de la sociedad civil y del Gobierno, quienes aprobaron un conjunto de políticas de Estado que constituyen un Acuerdo Nacional, que permita crear trabajo digno y productivo para todos; garantizar que todo peruano tenga pleno acceso a la salud, a la educación y la cultura; y construir un Estado al servicio de las personas. En este mismo sentido, se plantearon lineamientos de política a la luz del análisis de los problemas sanitarios priorizados (Ministerio de Salud, 2002).

Para abordar el problema, la política formuló estrategias enfocadas en la promoción de la salud, prevención de la enfermedad y desarrollo de un sistema de vigilancia epidemiológica con base intersectorial de importancia regional y nacional. En cuanto a la implementación, existen acciones de promoción de la salud y prevención de enfermedades que buscan evitar efectos adversos que puedan conllevar a la muerte materna, teniendo como finalidad salvaguardar la salud de las mujeres. Por otro lado, la gestión se desarrolla en todos los niveles de atención de salud, que va desde el nivel primario de atención hasta el nivel especializado y de mayor complejidad.

De la misma manera, en el primer momento se formularon objetivos para promover conductas y estilos de vida saludables en todos los grupos etarios de la población, dando especial tratamiento al grupo de mujeres en edad fértil como prioridad la MM, planteándose como meta reducir significativamente a menos de $100 \mathrm{MM}$ por 100. $000 \mathrm{NV}$, principalmente en los sectores rurales más pobres.

En este sentido, las estrategias empleadas por el MINSA para mejorar la salud materna están enfocadas a: i) participación y control ciudadano, ii) adecuación de la estructura del MINSA para ejercer la concertación y conducción sectorial, iii) compromiso de los recursos humanos con los objetivos sectoriales, iv) diálogo sectorial e intersectorial, v) descentralización del sector, vi) reorientación del financiamiento, vii) agenda compartida y concerta- ción con la cooperación técnica internacional y viii) establecer políticas regionales andinas comunes.

\section{Segundo momento histórico}

Antes de desarrollar el Plan Concertado de Salud, es necesario mencionar el "Acuerdo de Partidos Políticos en Salud", quienes tuvieron una intervención relevante en la formulación e implantación de políticas en salud, fortaleciendo el trabajo desarrollado en el Acuerdo Nacional. Es así, que el año 2006 diversos partidos políticos asumieron el reto de adoptar acuerdos por consenso con relación a la salud pública, como expresión del derecho a la salud de peruanos y peruanas, traduciéndose en políticas sanitarias a aplicarse en el próximo período de gobierno 2006-2011 en beneficio de la población, avanzando sobre lo ya construido en el Acuerdo Nacional.

Como expresión del trabajo realizado, los representantes de salud de los partidos políticos aprobaron 65 líneas de acción en salud para el período de gobierno 2006-2011, en las áreas de reforma del sector salud que parte desde el aseguramiento universal en salud, financiamiento en salud, descentralización del sector, participación ciudadana, acceso y disponibilidad de medicamentos. Asimismo, pone énfasis en los problemas de salud Materna, salud infantil, VIH-SIDA, malaria y TBC. Dicho acuerdo fue suscrito por las direcciones políticas de 16 partidos políticos y fue entregado para su ejecución al Ministerio de Salud y para su seguimiento a la Defensoría del Pueblo, al Acuerdo Nacional y al Foro Salud.

\section{Tercer momento histórico}

En este momento se desarrolla el Plan Nacional Concertado de Salud (PNCS), formulado e implementado en el gobierno de Alan García Pérez (período, entre el 28 de julio de 2006 y el 28 de julio del 2011). Este proceso ha marcado un hito en el camino de la concertación Estado - Sociedad Civil, ya que fue la primera vez que se realiza una consulta y discusión de una propuesta de plan nacional de salud en todo el país, con participación del Gobierno Nacional, Regional, Local y la Sociedad Civil. Este plan constituye un instrumento de gestión y de implementación de las políticas de salud respecto a las prioridades sanitarias del país y de las principales intervenciones que tienen como objetivo mejorar el estado de salud de la población. El PNCS planteó sus objetivos y metas de salud en el mediano y largo plazo (2007-2020), ordenando y optimizando los roles de todos los actores del 
sistema de salud, oenmarcado en los Objetivo de Desarrollo del Milenio (ODM), el acuerdo nacional y el consenso de partidos políticos en salud.

A la luz de lo mencionado, el PNCS formuló lineamientos que garanticen y fortalezcan la política de salud, haciendo hincapié en la i) atención integral de salud a la mujer y el niño privilegiando las acciones de promoción y prevención, ii) vigilancia, prevención y control de las enfermedades transmisibles y no transmisibles, iii) aseguramiento universal, iv) mejoramiento progresivo del acceso a los servicios de salud de calidad, v) desarrollo de los recursos humanos, vi) medicamentos de calidad para todos/as vii) financiamiento en función de resultados, viii) desarrollo de la rectoría del sistema de salud, entre otros.

Asimismo, en el lineamiento de política número uno incluye la atención integral de la salud sexual y reproductiva de la mujer, en todos los servicios de salud de manera integral considerando el enfoque de género y la necesidad de adecuar culturalmente los servicios en los ámbitos donde sea necesario respetando sus derechos sexuales y reproductivos, priorizando la promoción del parto institucional en el país, y fortaleciendo las intervenciones de planificación familiar, atención integral de las adolescentes y reducción de las muertes maternas.

Por otro lado el PNCS, formuló objetivos sanitarios, que se focalizaron en reducir la $\mathrm{MM}$, reducir el embarazo en adolescentes, reducir complicaciones del embarazo, parto, puerperio e incrementar el parto institucional en zonas rurales, y ampliar el acceso a la planificación familiar. Por su parte, las metas propuestas con horizonte temporal al año 2011 con una reducción de la MM de 185 a 120 por $100.000 \mathrm{NV}$ y para el 2015 se habrán reducido a 66 por $100.000 \mathrm{NV}$ la MM. De la misma manera, se formularon estrategias encaminadas a incrementar la cobertura de atención prenatal, del parto institucional en zonas rurales y dispersas e introducir métodos altamente eficaces para prevenir los riesgos relacionados al embarazo y al parto.

\section{Cifras de mortalidad materna en la República de Perú}

La mortalidad materna en el Perú viene exponiendo una tendencia decreciente en las últimas décadas, sin embargo en el contexto latinoamericano se sitúa entre los países con niveles más críticos. En este marco, de acuerdo a los datos de la Encueta Demográfica de Salud Familiar (ENDES) de 1996, la razón de mortalidad materna estimada para el país fue de 265 por 100.000 nacidos vivos, el mismo indicador para el año 2000 y con el uso de la misma metodología de medición evidenció una reducción cercana al 30 por ciento (Razón MM 185.000 por $100.000 \mathrm{NV}$ ) (Instituto Nacional de Estadística e Informática, 2000). Tal estimación permite afirmar que alrededor de 1.100 mujeres mueren anualmente en el Perú por causa materna, o dicho de otro modo cada día tres mujeres peruanas fallecen por causa relacionada al embarazo, parto y puerperio (Instituto Nacional de Estadística e Informática, 2000).

Así pues, las elevadas cifras de mortalidad materna han tenido un comportamiento estacionario hasta mediados de la década de los 90, cuando se inicia una tendencia decreciente que si bien no revierte la gravedad de la situación muestra una evolución favorable, reduciéndose de 400 a 185 por $100.000 \mathrm{NV}$ en el país (la última cifra corresponde al año 2000, ENDES), datos que se exponen en el gráfico № 10 . A pesar de ello, en el ámbito de América del Sur, la mortalidad materna en el Perú es la segunda mayor después de Bolivia, y la tercera de América Latina después de Haití (OPS, 2002), siendo el promedio latinoamericano de 130 por cada 100. 000 nacidos vivos (UNICEF, 2008).

Por consiguiente, la mortalidad materna en el Perú es elevada, tanto en el contexto latinoamericano y sudamericano, reflejándose las barreras económicas, culturales y geográficas. Asimismo, las inequidades y la deficiencia del funcionamiento de los servicios de salud como un sistema integrado. Bajo este contexto, un hecho fisiológico como la maternidad puede convertirse en una condición de riesgo para la vida de las mujeres (CEPAL, 2005)

En el gráfico presentado, se aprecia que los departamentos de Huancavelica y Puno con porcentajes de pobreza mayores en comparación a los demás departamentos, que se correlaciona positivamente con las mayores tasas de MM; si los comparamos con el departamento de Ica se observa un menor porcentaje de pobreza y que se relaciona con una menor tasa de MM, confirmando que las mayores tasas de MM se encuentran en lugares de mayor pobreza.

La muerte materna es una de las expresiones más claras de las condiciones de desigualdad que afectan principalmente a las mujeres en condiciones de vulnerabilidad social y económica; las mujeres que mueren por causas relacionadas al embarazo, parto y puerperio, son por lo general mujeres de más bajos ingresos, con menor escolaridad y que afrontan mayores dificultades de distinta índole en el acceso a los servicios de salud reproductiva. 
Figura 1: Tasa mortalidad materna, Perú 2000

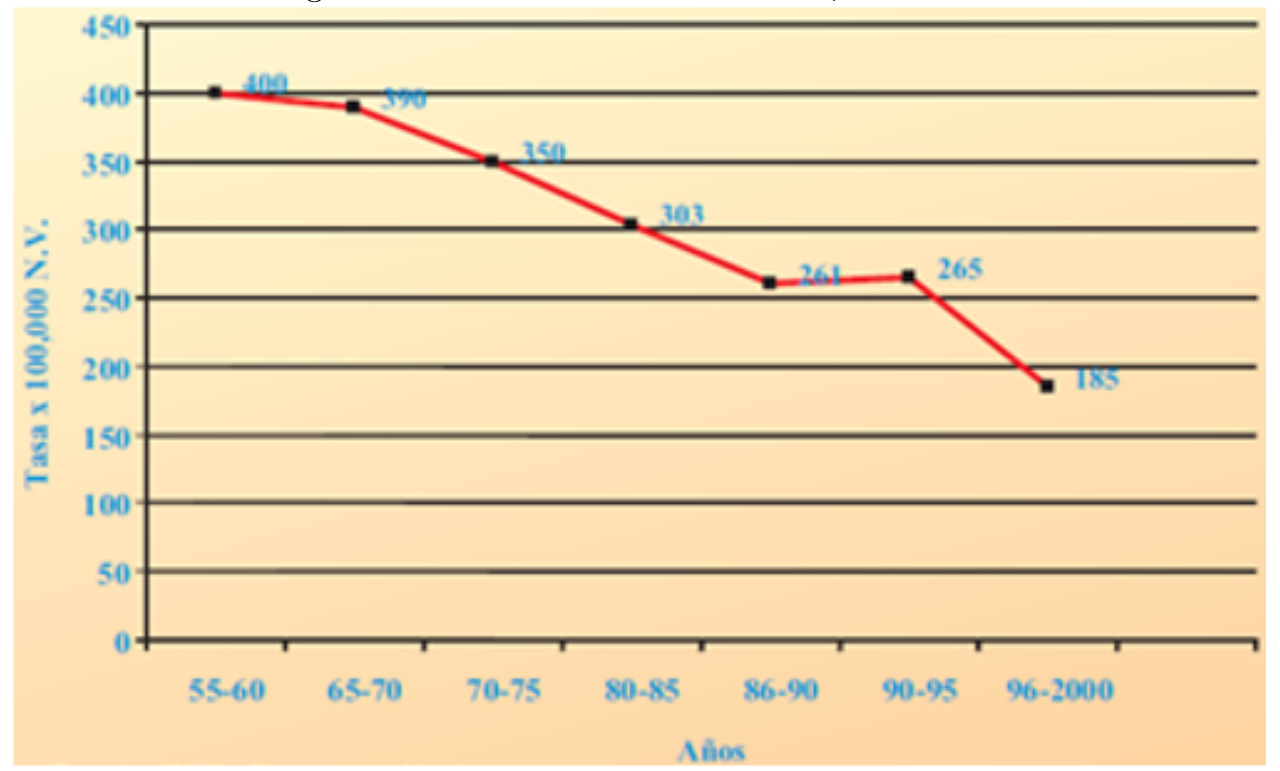

Fuente: Instituto Nacional de Estadística e Informática - Encuestas Nacionales (2000)

Figura 2: Muerte materna y pobreza según Departamentos, Perú 2002

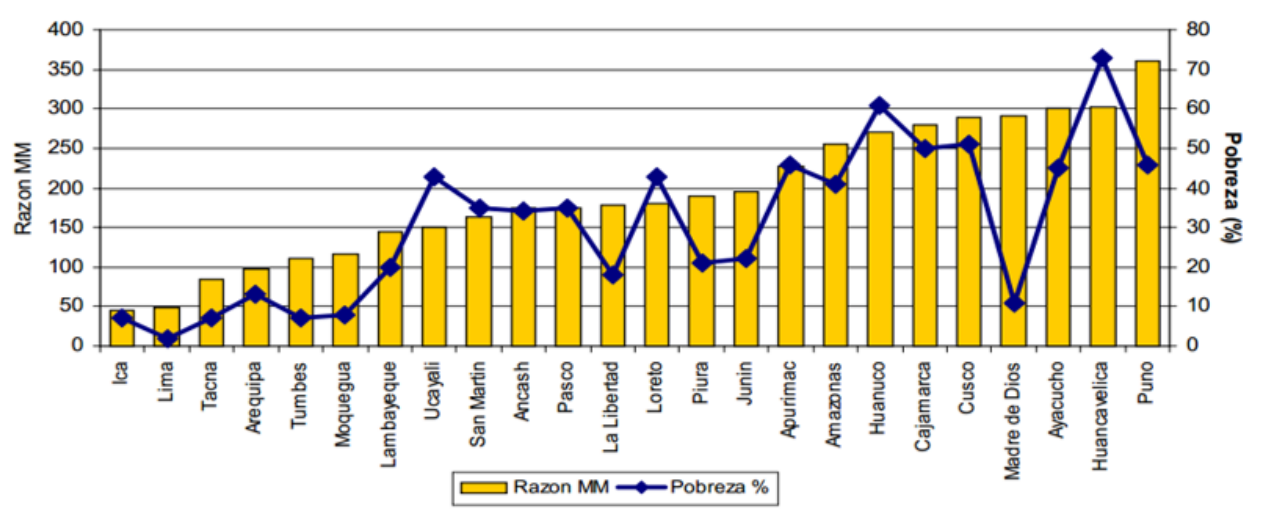

Fuente: Instituto Nacional de Estadística e Informática - Dirección General de Epidemiología (2002) 


\section{Dimensión diseño organizacional}

En consecuencia, el MINSA no ejerció liderazgo en el sector salud, contribuyendo a la fragmentación intrasectorial; su organización interna estuvo debilitada por la presencia de programas y proyectos financiados con cooperación externa que actuaban paralelamente a las estructuras formales de dirección, planificación y administración del nivel central y de las direcciones regionales (Ministerio de Salud, 2002).

Por ello, el Estado peruano implantó la descentralización e integración regional de salud, para hacer frente a la fragmentación del sector salud, donde los equipos de trabajo puedan desarrollar labores coordinadas con las Direcciones Regionales de Salud (DIRESAS), con el propósito de fortalecer su rol como única autoridad en salud en cada uno de los Gobiernos Regionales, sin resultados congruentes hasta la actualidad. Asimismo, ha impulsado el enfoque de interculturalidad, con la atención de parto y con instalaciones de casas de espera en las cercanías a los establecimientos de salud dispersos. Sin embargo, la distribución de las casas de espera sigue sin ser uniforme impidiendo el acceso de las mujeres a la atención de salud y a la atención obstétrica de urgencia que necesitan (Informe de gestión del Ministerio de Salud, 2007).

En la misma línea se implantaron políticas y programas de salud para los pueblos indígenas, con avances significativos en la legislación nacional, normativa específica sobre salud y población indígena que van desde el reconocimiento del derecho a la salud como bien individual, mencionando a las poblaciones indígenas como grupos prioritarios, hasta una legislación que reconoce y promueve los derechos colectivos de medicinas tradicionales (América Latina: Avances y desafíos de la implementación del Programa de Acción de El Cairo, 2010).

El Estado peruano ha diseñado e implementado estrategias como la afiliación de las mujeres gestantes al Seguro Integral de Salud (SIS), aplicando el enfoque de interculturalidad en la atención de parto y las casas de espera en funcionamiento en todo el país, cercanas a los establecimientos de salud (Informe de gestión del Ministerio de Salud, 2008).

El SIS emerge con el propósito de encarar el problema de fragmentación del Sistema de Salud, que persiste en tres estancos: i) el MINSA y las Direcciones Regionales de Salud (DIRESAS) que posee la red de establecimientos públicos más grande del país y que atiende, principalmente a la población de los quintiles I, II y III de pobreza (los de mayor pobreza); ii) la Seguridad Social que es la segunda red de mayor cobertura en el país pero exclusi- vamente destinada a la población asalariada y sus familiares, y iii) la Sanidad de las Fuerzas Armadas y las Fuerzas Policiales, con redes de menor extensión y cobertura (Estrategia de Cooperación Técnica OPS/OMS, 2010).

También incorporó nuevos programas estratégicos como el Presupuesto Por Resultados (PPR), como una nueva herramienta moderna de Gestión para lograr resultados en la población necesitada, que parte con una visión integrada de planificación y presupuesto, y la articulación de acciones y actores para la consecución de resultados (Informe de gestión del Ministerio de Salud, 2009).

\section{Dimensión de comunicación}

La comunicación intrasectorial, se caracteriza por tener trámites engorrosos, que obstaculizan la adquisición de insumos de manera oportuna como medicamentos, métodos anticonceptivos, materiales, entre otros; sumado a ello, los tiempos prolongados en el proceso de adquisiciones de bienes y servicios, que repercute en la oportuna realización de actividades programadas (Informe de gestión del Ministerio de Salud, 2009; Informe de gestión del Ministerio de Salud: I Semestre, 2010).

El MINSA, creo el área de Defensoría de la Salud y Transparencia del Ministerio de Salud, denominada Información de Salud (INFOSALUD), que brinda orientación y consejería en salud integral, información institucional y aseguramiento universal; atiende quejas sobre la atención en los diferentes establecimientos de salud del MINSA; y garantiza a la ciudadanía acceder gratuitamente a la comunicación con un profesional, cumpliendo con la misión de brindar un servicio gratuito, oportuno, permanente y de calidad, utilizando las tecnologías de la información y comunicación. Asimismo, dispone de diversas fuentes de información nacionales e internacionales que han permitido desarrollar un diagnóstico aproximado de las brechas en salud a nivel nacional; sin embargo, no se dispone de un sistema de información permanente para una eficiente toma de decisiones, a pesar de contar con estrategias como INFOSALUD, debido a la inaccesibilidad tecnológica de establecimientos de salud y poblaciones periurbanas y rurales (Informe de gestión del Ministerio de Salud, 2009).

El MINSA no ha cumplido durante décadas un rol de liderazgo en la formulación de políticas de salud intersectoriales. Existe una distribución de establecimientos y recursos humanos con criterios de priorización exclusivamente de cada sub-sector, lo cual lleva a formas diferentes y contradictorias de organización regional y local; existe también cierto 
divorcio entre la organización del sub-sector MINSA y la organización política del país a nivel de regiones, departamentos, provincias y distritos, donde el compromiso de los gobiernos regionales y locales es mínimo en el apoyo de las acciones y estrategias para disminuir la MM (Ministerio de Salud, 2002; Informe de gestión del Ministerio de Salud, 2009).

Desde el año 2000 el MINSA aprueba la Resolución Ministerial la Historia Clínica Materno Perinatal y su Aplicativo Analítico de Indicadores de Producción y Calidad de Servicios (SIP2000), con el fin de brindar una herramienta que sirva para fortalecer los procesos de gestión local y estandarizar los indicadores de calidad de atención en la prestación de salud brindada al binomio madre niño; a fin de contar con un sistema que amplíe la capacidad de obtención de datos y genere información útil para optimizar la atención de la madre y el niño (Avanzando hacia una maternidad segura en el Perú: Derecho de todas las mujeres, 2006).

La implementación de los datos estandarizados mediante los aplicativos informáticos que actualmente se encuentran funcionando en el nivel nacional, permitió compartir la información -codificada en una misma forma en todo el sector- en bases de datos, en este sentido la toma de decisiones será en forma global y no parcializada en forma oportuna, correcta y acordes con las políticas del sector salud (Informe de gestión del Ministerio de Salud, 2007).

El MINSA, no cuenta con un sistema efectivo de referencia y contrareferencia, que incluya medios de transporte adecuados que asegure la participación y disponibilidad de redes de apoyo informadas sobre signos de alarma durante la gestación, parto y puerperio (Comisión Económica para América Latina y el Caribe, 2004).

La comunicación sanitaria en la República de Perú, está en función a intervenciones importantes para mantener a la población informada sobre los cuidados de la salud, para ello, se desarrollaron campañas a favor de la maternidad saludable y segura, así como la promoción de medidas anticonceptivas seguras como los Anticonceptivos Orales de Efectivo (AOE), consiguiendo el respaldo para la distribución gratuita del método, con lo cual se disminuyó las posibilidades de muertes por embarazos no deseados y abortos clandestinos; asimismo, las acciones de difusión de mensajes e información a través de los medios de comunicación (radio, diarios, televisión e internet), relacionada a la salud materno neonatal, estuvieron enmarcadas en la promoción de hábitos saludables en la gestante, incidiendo en los controles prenatales, nutrición, entre otros; todas las actividades descritas se ha concluido en la elaboración de la Directiva Sanitaria de notificación e investigación para la vigilancia epidemiológica de la MM, lo que fortaleció el subsistema de vigilancia garantizando la calidad de la información (Informe de gestión del Ministerio de Salud: I Semestre, 2010).

\section{Dimensión recursos humanos y financieros}

Recursos humanos con capacidades y habilidades acorde a las necesidades del sistema de salud, donde el MINSA implemento programas de fortalecimiento de servicios de salud, área de capacitación y apoyo a la gestión. Así como el fortalecimiento de profesionales de la salud, en competencias de gestión para la atención integral de salud (Desafíos de la gestión de los recursos humanos en salud, 2005; Informe de gestión del Ministerio de Salud, 2008).

En el año 2000, sólo en el $20 \%$ de los partos se contaba con la presencia de un trabajador sanitario capacitado, mientras que en zonas urbanas la cifra era del $69 \%$; siendo las capacidades uno de los factores más estrecha y universalmente asociado con la reducción de la morbi - mortalidad materna, siendo la atención del parto por personal calificado, un eje que contribuye a evitar complicaciones y/o al manejo adecuado de las complicaciones (Salud materna y neonatal: Estado mundial de la infancia, 2009).

Asimismo, existe una débil capacidad técnica en auditoría de la calidad de la atención en salud de los profesionales en los órganos desconcentrados; siendo la causa principal el escaso compromiso con la institución y la idea errónea de la auditoría punitiva (Informe de gestión del Ministerio de Salud: I Semestre, 2010).

La República de Perú, ha experimentado un éxodo de profesionales de la salud y crecientes corrientes de emigración de personal calificado hacia los países desarrollados, que han tenido fuertes efectos negativos en la disposición de personal calificado (UNFPA/EAT: El derecho a la salud y los objetivos de desarrollo del milenio, 2004).

La disponibilidad de profesionales especializados es indispensable para disminuir los riesgos de la MM, mientras el acceso a este servicio es muy limitado para las mujeres de bajo nivel educativo y económico; existiendo una alta relación entre la mortalidad materna y la atención institucional del parto, sólo 58 de cada 100 partos son atendidos en servicios de salud, con marcadas diferencias entre las zonas urbanas y rurales (Mujer y Desarrollo: Caso Perú - CEPAL, 2004). La distribución del personal de salud en la circunscripción territorial del Estado peruano, es indicada por el centralismo 
e inequidad en su distribución de recursos humanos (La Salud en las Américas, 2002).

El Fortalecimiento de desarrollo y la gestión de los recursos humanos en salud, es uno de los grandes problemas que afronta la realidad sanitaria peruana, siendo la inadecuada distribución del personal asistencial, concentrándose en las capitales de departamentos y grandes ciudades (Informe de gestión del Ministerio de Salud, 2007).

A pesar de evidenciar un avance significativo con respecto a inicios de la década de 1990, los indicadores desagregados de partos institucionales (ocurridos en establecimientos de salud y atendidos por personal profesional de la salud) reflejan la persistencia de las enormes inequidades que permean el país (Hacia el cumplimiento de los objetivos de desarrollo del milenio en el Perú: Un compromiso del país para acabar con la pobreza, la desigualdad y la exclusión, 2004).

El Estado peruano inició proceso de nombramiento de $15 \%$ del total de profesionales de la salud no médicos cirujanos, contratados por locación de servicio a nivel nacional, lo que significa una inversión anual superior a los S/. 24.500.000,00 (S// CL 4537037 037.03) que permitirán la incorporación a la carrera administrativa a unas 1.495 personas (Informe de gestión del Ministerio de Salud, 2007).

El Programa Salud para Todos, focalizado a empleadores que brindan sus servicios en los establecimientos de primer nivel por contratos renovables de 90 días, sin derecho a vacaciones ni a beneficios sociales, esta modalidad laboral significa aproximadamente la sexta parte de los recursos humanos del MINSA (Lineamientos de política sectorial, 2001).

EL nivel remunerativo de los profesionales de la salud con régimen de Contrato Administrativo de Servicios (CAS) son desiguales, ocasionando inestabilidad laboral del personal capacitado en las regiones del territorio peruano (Informe de gestión del Ministerio de Salud, 2009).

Se gestiona la meritocracia, por intermedio de la Alta Dirección, en el proceso de la selección y elección de Directores Generales de Institutos Nacionales y Hospitales del Ministerio de Salud, siendo aún prematuro cimentar esta iniciativa (Informe de gestión del Ministerio de Salud, 2007).

La Oficina de Ingreso y Escalafón ha logrado la actualización del $90 \%$ de los Legajos de los servidores y funcionarios de la Administración Central de salud; pese al constante movimiento que generan las diferentes acciones del personal de recursos humanos, con el propósito de ascender en la carrera funcionaria (Informe de gestión del Ministerio de
Salud, 2008).

El MINSA, ha establecido un mejor control de las acciones de desplazamiento del personal de salud que se efectúan en la Administración Central, logrando una cobertura del $100 \%$ con porcentaje perfecto de lo programado, con el fin de evitar plazas vacantes en los establecimientos de salud y garantizar una permanente atención médica (Informe de gestión del Ministerio de Salud, 2008).

El problema de gestión más importante es referente a los recursos humanos, en este terreno la política aplicada durante la década pasada no ha tenido una propuesta clara desde el principio, ha oscilado entre una propuesta liberal a ultranza y la contratación flexible de personal en el mercado de trabajo, y otra propuesta de reconversión de los recursos humanos existentes mediante la capacitación y selección en el propio trabajo (Lineamientos de política sectorial, 2001).

El presupuesto por Resultados como fuente principal de financiamiento no se ha ajustado a las necesidades de las regiones, para ejecutar las actividades de la Estrategia Sanitaria de Salud Sexual y Reproductiva en especial para el fortalecimiento de la capacidad resolutiva de los Establecimientos de Salud (EESS) (Informe de gestión del Ministerio de Salud, 2009). La asignación del gasto en salud, es destinada a actividades de promoción, prevención y prestación del servicio de salud, incluye gastos de administración, capacitación de los recursos humanos y a la investigación aplicada en salud (Estrategia de Cooperación Técnica OPS/OMS Perú, 2010).

La oficina de presupuesto, en el marco del proceso de descentralización, condujo la identificación y cuantificación de los recursos presupuestales programados para el año fiscal 2009 relacionados a las funciones transferidas y la verificación de la existencia de los correspondientes créditos presupuestarios en el Presupuesto Institucional del Pliego 011 Ministerio de Salud, la transferencia presupuestal incorporo los presupuestos institucionales de 25 Gobiernos Regionales (Informe de gestión del Ministerio de Salud, 2009).

El presupuesto asignado por el SIS para la atención materna se ha ido incrementando progresivamente en los últimos años, donde el acceso a los servicios de salud ha mejorado por intervenciones del SIS y la Estrategia Crecer, pero aún persisten problemas en la calidad de los servicios prestados, así como en los niveles de resolución de establecimientos de salud ubicados en las zonas rurales y peri urbanas. (América Latina: Avances y desafíos de la implementación del Programa de Acción de 


\section{El Cairo, 2004).}

La trasferencia financiera, se realizó con un monto de treinta y cinco millones novecientos cuarenta y cuatro mil doscientos setenta y cinco y 00/100 nuevos soles (S// CL 6656347222.2 ) a ocho Gobiernos Regionales y dieciséis Gobiernos Locales para la ejecución de proyectos comprendidos en el marco del Aseguramiento Universal y proyectos priorizados de salud, en atención al D.U. № 114-2009-EF, lo cual se encuentra enmarcado en la política de mejoramiento de la oferta de los servicios de salud, redundando en una mayor capacidad de brindar servicios con calidad y oportunidad, especialmente en lugares cuyo acceso geográfico no permite una referencia inmediata (Informe de gestión del Ministerio de Salud, 2009).

\section{Dimensión negociación}

La política sectorial requiere una estrecha coordinación con los diferentes subsectores de salud, por lo cual el MINSA promueve permanentemente la concertación sectorial y también intersectorial; un eje de esta orientación estratégica es la conformación del Consejo Nacional de Salud (CNS), como ente concertador del Sistema Nacional Coordinado y Descentralizado de Salud (Lineamientos de política sectorial, 2001).

El MINSA, trabajó en la resolución de conflictos laborales reduciendo en un $37 \%$ los conflictos laborales en el Sector Salud, con relación al 2004 y 2005, dado que en las Mesas de Diálogo instaladas se aplicó como estrategia la negociación integrativa, para la atención de las demandas laborales de los gremios sindicales, (Federación Médica, Sindicato de Enfermeras y Obstetras) (Informe de gestión del Ministerio de Salud, 2007).

El MINSA realizó esfuerzo con otros sectores, a través del Programa Estratégico (Salud Materno Neonatal), cuyo propósito es ampliar la cobertura de atención prenatal y del parto institucional en zonas rurales y dispersas e introducir métodos eficaces para prevenir los riesgos relacionados al embarazo y al parto (Informe de gestión del Ministerio de Salud, 2007).

En el marco del convenio con el Ministerio de Educación, se fortaleció el trabajo intersectorial para el desarrollo de la promoción de la salud en las Instituciones Educativas, elaborándose el documento técnico identificación de temas de salud en los textos escolares de los niveles de Inicial, Primaria y Secundaria, así como la Dirección General de Promoción de la Salud, quien brindo asistencia técnica para la Implementación del Programa de Promo- ción de la Salud en Instituciones Educativas (Informe de gestión del Ministerio de Salud: I Semestre, 2010).

\section{Dimensión innovación}

Se renovó el $45 \%$ de los equipos de cómputo de las dependencias de la administración central del MINSA, por obsolescencia tecnológica; también, intervenciones de soporte técnico informático y de telecomunicaciones a las diferentes dependencias de la Sede Central del Ministerio de Salud, con la finalidad de dar sostenibilidad y soporte al proceso de gestión del sistema de salud (Informe de gestión del Ministerio de Salud, 2007).

Equipos de gran complejidad, adquiridos entre 1995 y 1998 para su red de establecimientos, no funcionaban, y se estimó que el $42 \%$ ya no funcionarían en 2002, aun teniendo una antigüedad máxima de cuatro a siete años; en consecuencia, el MINSA propuso como prioridad mejorar su capacidad de gestión técnica de equipos y dispositivos médicos, con énfasis en la planificación, adquisición, operación, uso, mantenimiento, renovación y regulación de tecnologías para fortalecer el procesos de atención médica garantizando cobertura y calidad (La Salud en las Américas, 2002).

La implantación del Registro Nacional de Establecimientos de Salud y Servicios Médicos de Apoyo a través de la aplicación Web RENAES, responsables de los establecimientos de salud a nivel nacional, ingresarán datos de sus establecimientos para que estos sean registrados oficialmente, permitirá que esta información esté disponible para cualquier persona, a través del Portal del MINSA, en el mismo instante en que es aprobado su registro (Informe de gestión del Ministerio de Salud, 2007).

Otras de las acciones realizadas fue la validación de la herramienta Encuestas para la medición de la satisfacción del usuario externo, utilizando la metodología SERVQUAL modificado en el marco del aseguramiento universal, en los servicios de consulta externa, hospitalización y emergencia del nivel hospitalario y consulta externa para el primer nivel de atención (Informe de gestión del Ministerio de Salud, 2010 - II Semestre).

Se priorizó las acciones de consolidación y programación de los recursos estratégicos (insumos y medicamentos básicos) a nivel nacional, alcanzando una ejecución financiera del $98 \%$ respecto a lo programado, lo cual ha permitido realizar la distribución de biológicos e insumos, así como el envío de remesas, logrando ejecutar las intervenciones sanitarias (Informe de gestión del Ministerio de Salud, 
2009).

La Salud en las Américas: Volumen II (2002) afirma que el Estado peruano ha desarrollado grupos de investigación, pero estos no se estructuran como un sistema nacional de investigación.

Es necesario que se norme el trabajo de promoción de la salud en apoyo a la salud de la madre y del recién nacido, donde los agentes comunitarios tienen una participación activa; en relación a ello la Dirección General de Promoción de la Salud, ha elaborado la Norma Técnica correspondiente, así como la elaboración de documento Técnico: Gestión Local para la implementación de las Casas Maternas, considerando el enfoque intercultural, para facilitar el acceso de las gestantes a la atención del parto institucional en el marco de la prioridad sanitaria de reducir la muerte materna y la Guía de Salud Sexual y Reproductiva por etapas de vida que establece las prácticas saludables por cada etapa de vida para ser difundidas y promovidas por el personal de salud (Informe de gestión del Ministerio de Salud, I Semestre, 2010).

\section{Política Pública en Salud Materna en la República de Chile}

\section{Momento histórico: Objetivos sanitarios pa- ra la década $2000-2010$}

Los objetivos sanitarios señalan las metas nacionales en salud que se deben alcanzaron en el año 2010 , constituyendo una referencia fundamental para establecer las intervenciones y acciones prioritarias que debe realizar por el sistema de salud chileno. Los objetivos para Chile recogen la experiencia internacional y entregan una visión de país, como elemento orientador, requiriendo la adaptación a las realidades locales. Definen metas concretas a alcanzar en áreas prioritarias, señalando las estrategias generales para el logro de cada objetivo, incluyendo intervenciones de eficacia comprobada en las áreas de promoción, prevención, tratamiento y rehabilitación, en un contexto de eficiencia en la entrega de servicios y de satisfacción de los ciudadanos (Gobierno de Chile, 2002). El resultado de este proceso, plantea que Chile enfrenta cuatro desafíos sanitarios: i) envejecimiento progresivo de la población, que involucra una creciente carga de patologías degenerativas de alto costo de atención médica; ii) desigualdades en la situación de salud de la población, que se traduce en una brecha sanitaria entre los grupos de población de distinto nivel socioeconómico; iii) responder adecuadamente a las expectativas legítimas de la población con respecto al sistema de salud, continuamente señalado como un elemento de insatisfacción para la sociedad chilena; y iv) resolver los problemas pendientes y mantener los logros sanitarios alcanzados (Gobierno de Chile, 2002).

Todos estos factores involucran cambios en la provisión de servicios sanitarios, tanto en la composición de los servicios, como en las formas de entregarlos, adecuándolos a los requerimientos técnicos sanitarios, así como a las exigencias de los usuarios, cada vez más conscientes y exigentes de sus derechos. De estos desafíos se derivan los cuatro objetivos sanitarios para la década: i) mejorar los logros sanitarios alcanzados; ii) enfrentar los desafíos derivados del envejecimiento y de los cambios de la sociedad; iii) disminuir las desigualdades; y iv) prestar servicios acordes a las expectativas de la población. Por tanto, el objetivo central del Sistema de Salud es mejorar la salud de la población, prolongando la vida y los años de vida libres de enfermedad y, al mismo tiempo, reducir las desigualdades en salud, mejorando la salud de los grupos más desfavorecidos de la sociedad (Gobierno de Chile, 2002).

En este sentido, se desarrolla el primer objetivo sanitario, donde está priorizada la salud de la mujer, donde se realizan intervenciones enfocadas a mejorar: i) salud reproductiva; ii) mortalidad fetal tardía; iii) calidad de la atención obstétrica; iv) morbimortalidad asociada al cáncer de cuello uterino; y v) climaterio.

Para fines del presente estudio se focaliza en la salud reproductiva, no dejando de ser vital las demás intervenciones, que permiten realizar acciones articuladas en beneficio de la salud de la mujer y que coadyuvan al logro de los objetivos y por ende a las metas planteadas; la salud reproductiva tiene como objetivo sanitario disminuir la MM en un $50 \%$, con tasa al 2010 de 1.2 por $10.000 \mathrm{NV}$ (Gobierno de Chile, 2002).

La razón fundamental de la disminución de la MM es por la mejora continua del acceso y la calidad de los servicios de salud reproductivos, impactando tanto en la sobrevida, como en el bienestar de las mujeres y sus familias; destacando los servicios y programa de planificación familiar, que comienzan a funcionar en Chile a mediados de la década del 60, impulsados por el gobierno como una forma de reducir la mortalidad materna por aborto. El impacto de esta iniciativa fue una reducción del $50 \%$ de la tasa global de fecundidad en 30 años. La mortalidad materna se redujo desde una tasa de 27.9 por 10.000 nacidos vivos en 1965 a 2 mil por diez mil nacidos vivos en 1998 (Gobierno de Chile, 2002). Un segundo aspecto relevante, ha sido la continua mejoría en el acceso al cuidado pre-natal, a través de la red de atención primaria, y a la atención hos- 
pitalaria del parto, que ha permitido reducir muertes evitables gracias a los avances tecnológicos de la atención obstétrica (Gobierno de Chile, 2002). Asimismo, en el objetivo sanitario ponen de manifiesto y para que la maternidad sea segura se requiere acciones simultáneas en tres frentes, i) reducir el número de embarazos no deseados y de alto riesgo; ii) reducir el número de complicaciones obstétricas y iii) reducir la tasa de mortalidad en las mujeres que presentan complicaciones.

Para manejar cada uno de los tres aspectos del problema, se debió aumentar la disponibilidad y el acceso a la información y a los servicios de planificación familiar para reducir substancialmente el número de embarazos, en especial los de alto riesgo y los no deseados. En este sentido se debe asegurar que todas las mujeres tengan acceso a servicios de salud durante el embarazo, el parto y el postparto, para así reducir la cantidad y la gravedad de las complicaciones obstétricas. Asimismo, se debe brindar información sobre la prevención y el tratamiento de las enfermedades durante el embarazo y sobre la detección precoz y su tratamiento cuando se presenten complicaciones. Finalmente, se debe proveer acceso a cuidados obstétricos esenciales a todas las mujeres que lo necesiten, con el objetivo de reducir la tasa de muerte cuando se presenten complicaciones.

En esta línea, para continuar el descenso de la mortalidad materna. Se necesita el esfuerzo de la sociedad en múltiples áreas: educación, condiciones de la vivienda, saneamiento básico, programas de inmunización, de alimentación complementaria, programas de salud especiales para las mujeres y los niños, atención profesional del parto, avances científicos y tecnológicos (Gobierno de Chile, 2002).

\section{Cifras de mortalidad materna en la Repúbli- ca de Chile}

La mortalidad materna en Chile ha presentado un sostenido descenso, como lo señalan las estadísticas a partir de 1961, año en que la tasa alcanzó a 318 por 100.000 nacidos vivos, disminuyendo en forma sistemática hasta llegar a dos dígitos el año 1978 (92 por 100 mil nacidos vivos), y continuando su descenso hasta ahora. En el año 1990, año base a partir del cual se definen las Metas de Desarrollo del Milenio, la razón de mortalidad materna en Chile ya era de 4.010 fallecimientos maternos por cada cien mil nacidos vivos; cifra que ha continuado descendiendo paulatinamente (Naciones Unidas y Gobierno de Chile, 2008).

Es así, que durante las últimas décadas se aprecia una favorable evolución de la mortalidad ma- terna correspondiendo a una de las tasas más bajas en América Latina. El año de 1990 correspondía a 40.0 por $100.000 \mathrm{NV}$, descendiendo a 17.1 por $100.000 \mathrm{NV}$ el año 2002 y manteniéndose en 16.9 por $100.000 \mathrm{NV}$ en el año 2008. Las principales causas de mortalidad materna son las complicaciones relacionadas con el embarazo y el aborto. Sin embargo, la baja sostenida de la mortalidad por aborto ha producido que en esta última década hayan sido más relevantes las complicaciones del embarazo. Recientemente las complicaciones del puerperio han llegado a ocupar el tercer lugar como causas de muerte materna en Chile (Naciones Unidas y Gobierno de Chile, 2005).

Cabe relevar que la disminución observada en la mortalidad materna se ha asociado al impacto del Programa de Salud Materna del Ministerio de Salud que incluye el control prenatal, el control del puerperio, la atención institucional del parto y la organización del sistema de atención en niveles crecientes de complejidad que permiten detectar precozmente y tratar por especialistas las patologías asociadas al embarazo (Naciones Unidas y Gobierno de Chile, 2005).

Asimismo, tiene un alto porcentaje de atención profesional del parto, sobre el $99 \%$ desde el año 1990, lo que asegura una buena atención perinatal y el consiguiente control de complicaciones que pudieran tener resultados mortales. Siendo para el año 1990 un porcentaje de $99.2 \%$, pasando a $99.7 \%$ el año 2000 (Naciones Unidas y Gobierno de Chile, 2005).

Desde estos elementos, es posible reconocer que en el país de Chile existe una favorable evolución en porcentaje de atención del parto por personal especializado que va desde un $45 \%$ en el año de 1952 , a $87 \%$ en el año de 1975 y un $99.8 \%$ para el año del 2009 (Naciones Unidas y Gobierno de Chile, 2011).

\section{Dimensión diseño organizacional}

La regulación sanitaria sobre los establecimientos de salud tanto del sector público y privado es responsabilidad estricta de las Direcciones de Servicios de Salud, y para establecimientos ubicados en la jurisdiccional territorial de las Direcciones de Servicio de Salud respectivamente (La salud en las Américas: Volumen I, 2002).

De este modo, se desarrolló un Sistema de Información Integral de Salud Intercultural (SIISI), Rakin Mongen Filu Lawen Pu che", en coautoría con Centro de Salud de Boroa Filu Lawen, con el objetivo de apoyar el registro de atenciones y de gestión, 
en sus aspectos interculturales y complementarios; el sistema abarca dos dimensiones por una parte la complementación de estadísticas de atención de salud convencionales occidentales con las atenciones propias de la medicina mapuche; y por otra parte, incorpora aspectos (variables) que permiten abarcar el concepto de salud integral mapuche, es decir, la salud interrelacionada con otros aspectos de la vida como la identidad, mantención de la cultura, vínculo con el mapu o territorio y actividades colectivas, entre otros (Informe de avance realizado CEPAL - ECLAC, 2010).

Organización Panamericana de la Salud, 2007 menciona que "el gobierno de Chile, implanto iniciativas de Redes Asistenciales basadas en la atención primaria de salud, teniendo como propósito desarrollar redes de salud a través del diseño de políticas para su coordinación y articulación, que permitan satisfacer las necesidades de salud de la población usuaria, en el marco de los objetivos sanitarios, con equidad, respeto de los derechos y dignidad de las personas" (21).

\section{Dimensión comunicación}

El MINSAL trabaja con equipos interdisciplinarios, donde participan una gama de profesionales de la salud juntamente con profesionales de otras áreas proporcionando una mirada amplia al abordar los diferentes temas de salud; es así que intervienen: Médicos, Matron/a, Enfermero/a, Kinesióloga, Geógrafo, Periodista, Ingeniero Químico, Bioquímico, Médico Veterinario, Sicóloga, entre otras profesiones. También, el MINSAL, desarrolla trabajo intrasectorial de manera coordinada y articulada desde diferentes dimensiones del sector salud, vale decir epidemiología, zoonosis, alimento, comunicaciones, urgencia ambiental, entre otros; todo ello fortalecido por el trabajo intersectorial del SEREMI, Servicio de Salud, Fuerzas de Armada, Ministerios y la sociedad civil (Naciones Unidas y Gobierno de Chile, 2005).

En la lógica de contribuir al fortalecimiento de un Sistema de Protección Social efectivo, que reduzca las inequidades, se desarrolla una nueva mirada que distingue - durante la gestación y los primeros años de vida de cada niño o niña - un espacio privilegiado para la consolidación de potencialidades que marcarán el futuro de las personas; surgiendo Chile Crece Contigo, iniciativa intersectorial que se constituye como un conjunto integrado de prestaciones, aspira a erradicar inequidades odiosas que pueden marcar la gestación, nacimiento y desarrollo inicial de los chilenos y chilenas, transformándose en verdaderas barreras para su evolución y éxito futuro (Manual de atención personalizada en el proceso reproductivo, 2008)

Reporte de Avance en el ODM 5 en América latina y el Caribe. Mejorar la Salud Materna (2010) menciona que:

\begin{abstract}
"Los índices menores de mortalidad materna que tiene la República de Chile, es en cierta medida debido a los esfuerzos emprendidos para mejorar la calidad del registro investigando las muertes de mujeres en edad reproductiva, se implementó el Sistema Nacional de Información Perinatal (SNIP), que es una estrategia del Departamento de Estadísticas e Información de Salud (DEIS) que apunta a establecerse como un repositorio de información nacional de Salud MaternoPerinatal y tiene como principal objetivo avanzar hacia la automatización de las estadísticas vitales, obteniéndolas a través del registro directo en las maternidades por profesionales de la salud. El SNIP es un sistema web, que permite dar salida a información en tiempo real a través de las extracciones de bases de datos, como mediante los reportes diseñados en base a las necesidades de los establecimientos de salud" (4).
\end{abstract}

Las necesidades de salud en el grupo de menor riesgo se resuelve, en general, en el primer nivel de atención (establecimientos de atención primaria); el grupo de alto riesgo en cambio, requiera ser atendido en niveles de mayor complejidad y capacidad resolutiva, sea ambulatorios (consultorio de nivel secundario) u hospitalarios, por personal especializado.

En la República de Chile, las actuales políticas de salud están centradas en las personas y con enfoque de determinantes sociales, que asumen el proceso de reproducción humana como un acontecimiento vital en las vidas de mujeres y hombres que da origen al nacimiento de una persona, única, destinada a jugar un rol irrepetible en el grupo familiar y en la sociedad en la que ha nacido; las intervenciones de promoción y prevención comienzan mucho antes del parto; por ello, los futuros padres y las familias deben de tener la posibilidad de crear las mejores condiciones para el niño o niña que desarrolle todas las potencialidades incluidas en su capital genético (Manual de atención personalizada en el proceso reproductivo, 2008).

\section{Dimensión recursos humanos y financieros}

UNFPA/EAT: El derecho a la salud y los objetivos de desarrollo del milenio (2004) afirma que: 
"El indicador de atención del parto es útil para identificar situaciones en que se encuentran países donde se aun necesitan realizar mejoras sustanciales; por su parte la República de Chile, posee una cobertura de un $100 \%$, de atención de parto por personal capacitado, encabezando la lista de países con menores rezagos en este indicador materno. Uno de los factores más estrecha y universalmente asociado a la reducción de la morbilidad y la mortalidad maternas es la atención del parto por parte de personal calificado (todo profesional de la salud: matronas, médicos, enfermeras), que contribuye a evitar complicaciones y a remitir los casos a otros servicios, entre ellos los de planificación familiar y el tratamiento de enfermedades de transmisión sexual" (153).

Con el fin de ofrecer oportunidades para que el personal de salud conozca mejor los cambios en la prestación de servicios de salud reproductiva, varias universidades han comenzado a ofrecer cursos de especialización o de posgrado, garantizando la formación de profesionales especializados (La salud en las Américas: Volumen II, 2002).

En los servicios de salud se encuentra la desregulación que atañe a la flexibilidad laboral, así como a la modificación de mecanismos corporativos que influyen sobre la conformación de los equipos de trabajo, la composición y ámbitos de las profesiones de salud y la autonomía profesional. La búsqueda de flexibilidad laboral como criterio esencial para disminuir los costos y crear condiciones de mayor competitividad es uno de los paradigmas vigentes en la gestión del personal en la economía en general y en el sector salud en particular (La salud en las Américas, 2002).

La asignación presupuestaria del Estado chileno, ha sido adecuada debido a su distribución de recursos entre proveedores y atenciones de salud de manera óptima, maximizando las ganancias en bienestar social; las ganancias contemplan al menos dos componentes: i) mejorías en el estado de salud de la población beneficiaria y ii) aumentos en el grado de protección financiera de las familias frente a eventos de salud (enfermedad); con eficiente asignación presupuestaria logra, una combinación óptima de disminuciones de morbilidad y mortalidad y, el aumento en la protección financiera de los hogares. Por su parte, el Ministerio de Hacienda da a conocer el Marco global de presupuesto de continuidad de los Servicios de Salud; con esa información, el MINSAL procede a asignar su presupuesto principalmente por líneas programáticas, tomando como base las asignaciones por programas presupuestarios, elaborados por distintos actores; esta forma de asignar los recursos trae como consecuencia que ciertos Servicios de Salud quedan, al final del periodo anual, con superávit presupuestario y otros con déficit sin dar cuenta de la necesidad específica de presupuesto de los Servicios de Salud, en consecuencia, esta asignación parcializada no permite realizar una mejor asignación basada en criterios de equidad y eficiencia, tendiendo a perpetuar las brechas (Hacia un presupuesto poblacional que reemplace el presupuesto histórico en el sistema nacional de servicios de salud: Propuesta metodológica y estimaciones, 2009).

Hacia un presupuesto poblacional que reemplace el presupuesto histórico en el sistema nacional de servicios de salud: Propuesta metodológica y estimaciones (2009) afirma que:

\footnotetext{
"Una distribución presupuestaria es equitativa cuando contribuye a reducir las brechas en el acceso a la atención de salud de las personas, las brechas de equidad corresponden a diferencias, entre individuos o grupos de individuos, en el acceso a la atención de salud. El gobierno chileno ayuda a reducir las diferencias asignando un mayor presupuesto allí donde hay mayores necesidades de salud, de modo de aumentar la disponibilidad de servicios y la calidad de la atención; a nivel territorial se expresa en asignar de manera prioritaria los recursos a aquellos Servicios de Salud que poseen mayores brechas de acceso y mayores necesidades sanitarias" (37).
}

Transferencias financieras están enfocadas hacia la Atención Primaria Municipalizada y otros montos menores a otros agentes financiados desde el nivel Gobierno Central, además del gasto corriente de los Servicios de Salud; este ejercicio permite dar cuenta del volumen global de recursos de que dispone una población determinada adscrita al territorio de un Servicio de Salud. No obstante, los montos asignados a la Atención Primaria municipalizada y los niveles secundario y terciario a cargo de los Servicios de Salud (Gobierno Central) se determinan hasta ahora en forma independiente; esto quiere decir lo que recibe una población determinada para financiar la Atención Primaria no guarda relación alguna con los recursos que recibe el Servicio de Salud para financiar la atención ambulatoria de especialidades y la atención hospitalaria y de emergencia. De esta forma, por mayor equidad en la asignación poblacional que se logrará con el sistema per cápita de la Atención Primaria municipalizada, el mecanismo actual de presupuesto histórico de los Servicios de Salud termina introduciendo, profundas inequidades en la asignación agregada poblacional (Hacia un presupuesto poblacional que reemplace el presupuesto histórico en el 
sistema nacional de servicios de salud: Propuesta metodológica y estimaciones, 2009).

\section{Dimensión negociación}

MINSAL, desarrolló estrategias para garantizar y proporcionar acceso a los jóvenes, para recibir atención en los servicios de SSR; no obstante, se ve dificultado por diversas razones como la resistencia de sectores conservadores, oferta insuficiente de servicios "amigables" en los establecimientos de atención primaria, jóvenes que no acuden a los centros establecidos para solicitar estos servicios (ODM, Segundo Informe, 2008).

ODM, Segundo Informe (2008) menciona que:

"Para el logro de las metas planteadas, es importante desarrollar un trabajo dirigido a los grupos más vulnerables, especialmente los jóvenes, a través de mecanismos adecuados y pertinentes como campañas educativas masivas y acciones específicas a nivel de la población escolar; para ello MINSAL, desarrollo estrategias en incentivar y mejorar la coordinación intersectorial, especialmente con el Ministerio de Educación, para el desarrollo de líneas de trabajo conjuntas. La propuesta gubernamental en sexualidad responsable, iniciativa intersectorial en desarrollo entre los Ministerios de Educación y Salud, Servicio Nacional de la Mujer (SERNAM), e Instituto de la Juventud (INJUV), se plantea como una de las estrategias para avanzar en el ámbito de la Salud Sexual y Reproductiva de este grupo, a través de modelos de gestión local al interior de las comunas" (115).

\section{Dimensión innovación}

En el 2001, se habían creado grupos, unidades $\mathrm{u}$ organizaciones dedicadas a la evaluación de las tecnologías de salud. En Chile, se trata de unidades del Ministerio de Salud cuya finalidad fue impulsar y coordinar la creación de redes articuladas de evaluación de tecnologías en salud en los distintos niveles del susbsector público, haciéndose cargo del mantenimiento de los equipos garantizando una atención con calidad (La salud en las Américas, 2002).Asimismo, cuenta con la Unidad de Evaluación de Tecnologías de Salud, encargada de apoyar técnicamente la identificación de los procesos más efectivos en función del costo para la atención de los problemas prioritarios de salud pública del país (La salud en las Américas, 2002).

A través de las TIC el MINSAL se incorporan nuevos programas nacionales denominados Salude y telemedicina; y el Estado tomó la decisión estratégica de implementar un sistema de información en salud para abordar de manera integrada el corazón de la gestión hospitalaria, centrado en la atención clínica de los pacientes. En ese sentido, se desarrolló un sistema de información en salud centrado en el usuario, que implementa la ficha clínica electrónica que contendrá el historial clínico del paciente -registro de atenciones, resultados de exámenes de laboratorio e imaginología, prescripción de fármacos, etc.- disponible desde cualquier centro de la Red de Salud, lo que asegura la continuidad en la atención, otorgando mayor calidad y seguridad a los pacientes (Salud y TIC - CEPAL - Unión Europea, 2010).

Uso racional de medicamentos: Una tarea de todos (2010) afirma que:

"El Ministerio de Salud de Chile, atendida la relevancia de los medicamentos dentro del sistema de salud público y privado, ha planteado en la Política Nacional de Medicamentos (Res. Ex. MINSAL № 512/2004) los lineamientos y directrices bajo las cuales se deberá desarrollar la actividad nacional en materia farmacéutica y dentro de éstos se encuentran las líneas de acción respecto de la Racionalidad en el Uso de Medicamentos, que estructuran una serie de actividades destinadas a lograr un uso racional de los mismos. En el desarrollo de estas líneas de acción y sus actividades correspondientes, es que el Ministerio de Salud ha decidido avanzar sobre la promoción del Uso Racional - de Medicamentos (URM) en todas sus áreas, para lo cual se han desarrollado documentos como el Manual de Selección de Medicamentos, la Guía para las Buenas Prácticas de Prescripción y un Marco Conceptual para la enseñanza sobre medicamentos y su uso racional. En esta misma línea de acción, y dando un paso hacia los procesos de educación del personal de salud, se coloca a disposición del mismo el presente documento, destinado a la entrega de conceptos básicos relacionados con los medicamentos que propiciarán una cultura de responsabilidad en torno a los mismos, así como su traspaso a la población en los actos de asistencia al paciente y entrega de medicamentos" (1).

La Salud en las Américas: Volumen II (2002) afirma que "el Estado, a través del Consejo Nacional de Ciencia y Tecnología, incentiva la investigación en salud, que se ha orientado más a las áreas de ciencias básicas y clínicas que a las de salud pública. También, la investigación en salud se realiza en las universidades y en los centros de investigación" (166). 


\section{Innovación en política de salud materna}

Las actividades que se realizan en el contexto del Programa de Salud de la Mujer, han sido siempre prioridad desde los inicios del Servicio Nacional de Salud que continúan siendo una de las bases de la atención en la población; las atenciones que se brindan constituyen desde mucho tiempo derechos básicos para las mujeres y se traducen en el contacto permanente entre el personal de salud y las usuarias. Por consiguiente dichas actividades tienen asegurada su continuidad en el tiempo, y es esperable dar cumplimiento integral a las metas planteadas en los Objetivos de Desarrollo del Milenio (ODM, Segundo Informe, 2008).

\section{Conclusiones}

\section{Dimensión de orientación general de política}

En la dimensión de orientación general de política, cabe destacar que la MM tuvo tratamiento en el caso de Chile muy anterior al de Perú, siendo el gobierno el principal actor en promulgar leyes que hicieron frente al problema sanitario. Dado que se trataba de una visión de largo plazo los gobiernos entrantes daban continuidad a la política implantada, además de fortalecerla con mecanismos favorecedores como los recursos financieros y humanos. Por otro lado, ambos gobiernos priorizaron las políticas de promoción y prevención en la salud materna, sin embargo Chile obtuvo mejores resultados y sigue siendo una de las principales políticas que ha incentivado desde las años noventa; por su parte Perú implantó este tipo de políticas a inicios del 2000, con resultados no muy alentadores. Finalmente, se observa que la historicidad institucional marca una diferencia en las políticas y repercute en la salubridad de la población.

\section{Dimensión de diseño organizacional}

En la dimensión diseño organizacional, se observa que los ministerios de salud que cuentan con una estructura organizacional segmentada y fragmentada presentan dificultades para articular sus acciones no logrando coberturas para la población, evidenciándose por lo tanto una mayor inequidad en el acceso a recibir atención médica. Por otro lado, la segmentación incentiva al centralismo de los ministerios imposibilitando una adecuada coordinación con los niveles de atención primario, en este sentido la coordinación funcional tanto del nivel na- cional y subnacional con marcada segmentación dificulta la toma de decisiones de forma eficiente, ya que la información con que cuentan los decisores no es lo óptimo, existiendo subregistros y pérdida de información relevante, por el contrario países que muestran estructura organizacional vertical y coordinada facilitarán la consecución de las coberturas en salud.

En este sentido, los ministerios de salud que no ejercen liderazgo dentro su sector siguen contribuyendo a la fragmentación y segmentación intrasectorial, haciendo que la implementación de las políticas en salud no logre alcanzar sus propósitos, perpetuando la insalubridad que se refleja en indicadores de la MM.

Por otra parte, las políticas y programas de salud implantadas y focalizadas en grupos vulnerables como los pueblos indígenas, son un claro avance en materia de salud apoyada por la legislación de los países, para disminuir las desigualdades y poder incrementar el acceso a los establecimientos de salud, con uso de técnicas de parto con adecuación cultural disminuyendo las MM y minimizando sus efectos lo que verá reflejado en su indicador.

\section{Dimensión de comunicación}

La comunicación es uno de los factores claves para garantizar una implementación de manera adecuada; en este sentido, sistemas de información robustos permitirán facilitar la implementación de la política en salud materna con decisiones basadas en evidencia, por el contrario la ausencia de sistema único de información obstaculizará la toma de decisiones, por lo que el alcance que pretende lograr la implementación de la política en salud materna sigue siendo insuficiente con lo que la política sigue siendo insuficiente.

En esta misma línea, tener acciones fortalecidas con otras instituciones, donde el trabajo articulado de manera intersectorial potencia las acciones de implementación en salud materna, observándose una mayor amplitud de sus logros en esta materia. En este mismo sentido, al existir desarticulación y duplicidad de funciones entre diversos órganos estatales que implementan políticas de salud redunda en la existencia de instancias débiles de coordinación que debilitan el trabajo intersectorial.

Por otro lado, si la comunicación es insuficiente, en el ministerio de salud se va instalando la separación en compartimentos estancos; y la implementación de la política no llega a cumplir con su propósito que es disminuir la MM a nivel de país, de regiones, departamentos, provincias y distritos, ya 
que las acciones y estrategias difieren significativamente. En este sentido, el rol rector del ministerio de salud, es implementar políticas que involucren a los gobiernos regionales, en el proceso de supervisión integral a nivel nacional, con la finalidad de promover la participación descentralizada.

\section{Dimensión de recursos}

Uno de los principales determinantes que se observa para que el sector salud pueda cumplir su misión es el desarrollo de los recursos humanos, resultando imprescindible el diseño de los perfiles de los servidores de salud con orientación estratégica y capacitación permanente.

Otro elemento relevante son los niveles de remuneración y las condiciones de trabajo ya que cuando esto no están no cumple con la expectativa del personal ocurre una alta rotación del personal capacitado, los que se alejan de las poblaciones rurales y periurbanas, ello afecta directamente no solo a la implementación políticas de salud, sino también a todas las políticas de salud.

Mejorar el acceso a la atención de parto por personal calificado, es clave para disminuir las complicaciones y las muertes maternas; por lo que es necesario contar con acciones que ayuden a mejorar la distribución y dotación de personal capacitado, dando prioridad a las regiones más pobres, focalizando en las acciones en la Atención Primaria de Salud, con asignación financiera para fortalecer el primer nivel de atención.

\section{Dimensión de negociación}

La negociación intrasectorial e intersectorialidad, es quien garantiza la viabilidad de estratégicas con mayor articulación de las redes sociales desde distintos niveles con la finalidad de potenciar las acciones para el logro de resultados de mayor impacto en la salud materna; desde este mismo modo facilitar la sinergias de acciones de los diferentes actores, es disminuir la fragmentación de las políticas en el proceso de implementación, el cual evita la dispersión de recursos con la proliferación y superposición de programas.

\section{Dimensión de innovación}

La innovación como dimensión en el proceso de implementación de la política, en la actualidad es uno de los factores que no se puede dejar de abordar, más aun en la línea de la salud que cada día las innovaciones y avances que permite actuar con mayor efectividad en las estrategias para disminuir la MM, todo esto impulsado desde los ministerios de salud, con ello facilita una mejor intervención con énfasis en la salud materna, para aminorar las brechas en el acceso a la atención de los servicios de la salud.

\section{Recomendaciones}

\section{Recomendaciones orientadas a mejo- rar la calidad}

Garantizar un seguimiento adecuado de la política del gobierno para la reducción de la mortalidad materna a fin de promover una planificación y rendiciones de cuenta en forma eficaz.

Reformular políticas para mejorar la capacidad de respuesta de los sistemas de salud, con abordaje intercultural, que garantice atenciones interculturales desde el embarazo, parto, puerperio y atención del recién nacido; respetando la cultura de la madre y optimizando la calidad de las atenciones.

Implementar políticas orientadas al mejoramiento y fortalecimiento de los sistemas de información, clave para el perfeccionamiento de las estadísticas vitales y del monitoreo de datos generados en el sistema de salud, garantizando una adecuada toma de decisiones basado en información fidedigna, con lo consiguiente generar impacto en la salud e la población.

Formular políticas para incrementar y hacer más progresivo el gasto corriente y de inversión en salud. Impulsar políticas que garanticen intersectorialidad e interinstitucionalidad, para establecer alianzas estratégicas mediante la articulación de redes sociales de distinto nivel institucional, comunitario o individual, que involucren a diferentes sectores y actores de la sociedad; para potenciar el logro de resultados reconociendo recursos activos de la comunidad no sólo incluye las fortalezas de cada individuo, sino también las asociaciones ciudadanas como las organizaciones de base, organizaciones comunales, iglesias, clubes, grupos culturales, escuelas, universidades, colegios profesionales, entre otros.

Formular políticas públicas y programas sociales compuestos con una orientación general que se nutra de las sinergias entre ellos; evitando la fragmentación de las políticas, la dispersión de los recursos y la proliferación y superposición de programas, y asegure su permanencia en el tiempo.

Implementar políticas para llevar a cabo inversio- 
nes adecuadas en infraestructura, equipos y aplicativos informáticos; así como en enlaces de telecomunicación capaces de soportar los sistemas de información que el sector salud requiere, para mejorar la capacidad de respuesta de los sistemas de salud para avanzar en equidad y extender la protección social en materia de salud materna.

\section{Recomendaciones orientadas a mejo- rar la cobertura}

Reforzar el proceso de coordinación en los órganos de línea de los ministerios de Salud, para consolidar la rectoría nacional y garantizar las condiciones necesarias para una adecuada implementación de políticas en salud materna.

Mejorar el rol rector del ministerio de salud, implementando políticas que involucren a los gobiernos regionales, en el proceso de supervisión integral a nivel nacional, con la finalidad de promover la participación descentralizada, la autoevaluación, la mejora en el desempeño y la transparencia en la gestión.

Reorientar y desarrollar trabajos sectoriales destinados a disminuir la segmentación y fragmentación del sistema de salud, perfeccionando su rol y funciones en nuevos escenario que plante la descentralización y el Aseguramiento Universal en salud; así pasar de un enfoque sectorial, a un sistema de salud integrado con visión intergubernamental que se capaz de articular e integrar el sistema de salud.

Incorporar políticas que reformulen las normas de salud relacionadas al aseguramiento universal, como la categorización y acreditación de EESS para el sector salud.

Impulsar políticas capaces de generar una regulación de los Sistema de Referencia y Contra Referencia (SRCR), siendo instancias que asegure la continuidad y oportunidad de la atención obstétrica, para coberturar la demanda insatisfecha optimizando los recursos de comunicación y transporte existente.

Formular políticas encaminadas a mejorar el acceso a atención de parto por personal calificado, clave para disminuir las complicaciones y las muertes maternas; por lo que se necesita políticas que ayuden a mejorar la distribución y dotación de personal capacitado, dando prioridad a las regiones más pobres y con mayor vulnerabilidad.

Promover el desarrollo de recursos humanos basados en la Atención Primaria de Salud, generando capacidades gerenciales y técnicas de los mismos.
Gestionar la asignación y ejecución de mayores recursos presupuestales orientados a disminuir la morbimortalidad materna, uno de los principales objetivos, priorizando las zonas o regiones donde se registran mayores índices de mortalidad materna, en coordinación con el sistema de vigilancia epidemiológica, a través de una ampliación constante de la cobertura nacional.

Diseñar políticas y programas de salud que articulen adecuadamente el conjunto de prestaciones e intervenciones de mayor impacto, según las características propias de las poblaciones a quienes se quiere beneficiar, tomando en cuenta su ubicación geográfica, su condición socioeconómica y su origen étnico, factores que muchas veces constituyen barreras para la eficaz puesta en marcha de las políticas de salud.

\section{Recomendaciones mixtas}

Desarrollar recursos humanos frecuentemente postergados, siendo imprescindible mejorar el perfil de los servidores del sector. Esta orientación estratégica buscará la capacitación permanente del personal de salud, a la vez que impulsará niveles adecuados de remuneración y condiciones de trabajo. Las principales políticas que se propone son: i) impulsar una adecuada política de gestión de los recursos humanos; ii) participación de los recursos humanos en la definición de los lineamientos de política sectorial; iii) capacitación permanente con los lineamientos de política; iv) promoción por sistema de méritos; v) remuneraciones idóneas de acuerdo a la responsabilidad; vi) incentivos familiares, personales y profesionales; y vii) fomento de la solidaridad en el desempeño y en la organización.

Tomando en consideración que existen situaciones nacionales que no permiten cubrir la inversión de la puesta en marcha de políticas y programas con fondos del presupuesto nacional, se recomienda la formulación de proyectos para acceder a fondos internacionales; tarea que requerirá la capacitación de equipos en el diseño de proyectos dentro de los ministerios de salud a nivel nacional, para llevar a cabo eficazmente las negociaciones con agencias de cooperación internacional e instituciones financieras.

Asignación financiera para fortalecer el primer nivel de atención e impulsando una mayor participación de la sociedad civil en el trabajo de sensibilización y autocuidado.

Garantizar una participación significativa de las mujeres en las decisiones sobre atención de la salud materna; asimismo, proporcionar a las mujeres 
información accesible sobre sus derechos sexuales y reproductivos y el derecho a la salud.

Impulsar políticas en planificación sanitaria y particularmente en planificación de provisión de insumos y medicamentos. Si bien, en este tipo de análisis se pone el énfasis en el financiamiento corriente y se hace la opción de formular un presupuesto prospectivo que satisfaga la oferta, la planificación de la provisión debe orientar las inversiones para garantizar que en el futuro la localización de los servicios y la distribución geográfica, se acerquen a las necesidades de la población. De lo contrario, la inercia de la inversión seguirá concentrada en las grandes ciudades que constituye una fuente de nuevas y más agudas inequidades.

\section{Referencias}

(2006). La Salud Neonatal en el Contexto de la Salud de la Madre, el Recién Nacido y el Niño para Cumplir los Objetivos del Milenio de las Naciones Unidas. $47 i i \frac{1}{2}$ Consejo Directivo de la Organización Panamericana de la Salud. Report, Organización Panamericana de la Salud and Organización Mundial de la Salud and USAID.

Comisión Económica para América Latina y el Caribe (2004). Las Metas del Milenio y la igualdad de género: el caso de Perú. Unidad Mujer y Desarrollo. Report.

Comisión Económica para América Latina y el Caribe, Organización Panamericana de la SaludOrganización Mundial de la Salud, UNICEF, United Nations Population Fund, y Fondo de Desarrollo de las Naciones Unidas para la Mujer (2009). Reunión de Expertos: Los determinantes de Mortalidad Materna en México y América Central: Hacia un Enfoque Multisectorial. Report.

de Administración y Políticas de Salud, I. (1997). Estudio de la mortalidad materna. Provincia de Mendoza, Años 1993-1994-1995. Report, Ministerio de Salud.

Gérvas, J. y Pérez, M. (2011). Rebajas sanitarias racionales en los tiempos de crisis económica. FCM. Medicina General. Equipo CESCA., Madrid. España.
Ministerio de Salud (2002). Lineamiento de política del sector salud: Perú, 2002-2012. MINSA, Lima.

Naciones Unidas (2000). Declaración del Milenio. Resolución A/RES/55/2. Report.

Naciones Unidas y Gobierno de Chile (2005). Los Objetivos de Desarrollo del Milenio: Primer Informe del Gobierno de Chile. Technical report, Santiago de Chile.

Naciones Unidas y Gobierno de Chile (2008). Los Objetivos de Desarrollo del Milenio: Segundo Informe Gobierno de Chile. Technical report, Santiago de Chile.

Naciones Unidas y Gobierno de Chile (2011). Objetivos de Desarrollo del Milenio: Tercer Informe de Gobierno de Chile. Technical report, Santiago de Chile.

Organización Mundial de la Salud, UNICEF, y FNUAP (2003). La tasa de mortalidad materna es desproporcionadamente alta en los países en desarrollo. Report.

Organización Mundial de la Salud, United Nations Population Fund, UNICEF, y Banco Mundial (1999). Reducción de la Mortalidad Materna. Declaración Conjunta. Report.

Organización Panamericana de la Salud (2007). Salud en las Américas. Technical report, Washington, D.C.

Ortún, V. (1996). Innovación en sanidad. AES, Barcelona.

Pérez Serrano, G. (2002). Investigación Cualitativa II. Técnicas de análisis de datos. Editorial La Muralla, España.

Rubio, V. O. (1996). La contribución de la economía de la salud a la reforma y gestión de los sistemas de salud. Universidad Pompeu Fabra, Barcelona, España.

Studin, I. (2000). Strategic Healthcare Management. Irwing Professional Publishing, Nueva York.

Yin, R. K. (2013). Case study research: Design and methods. SAGE publications. 